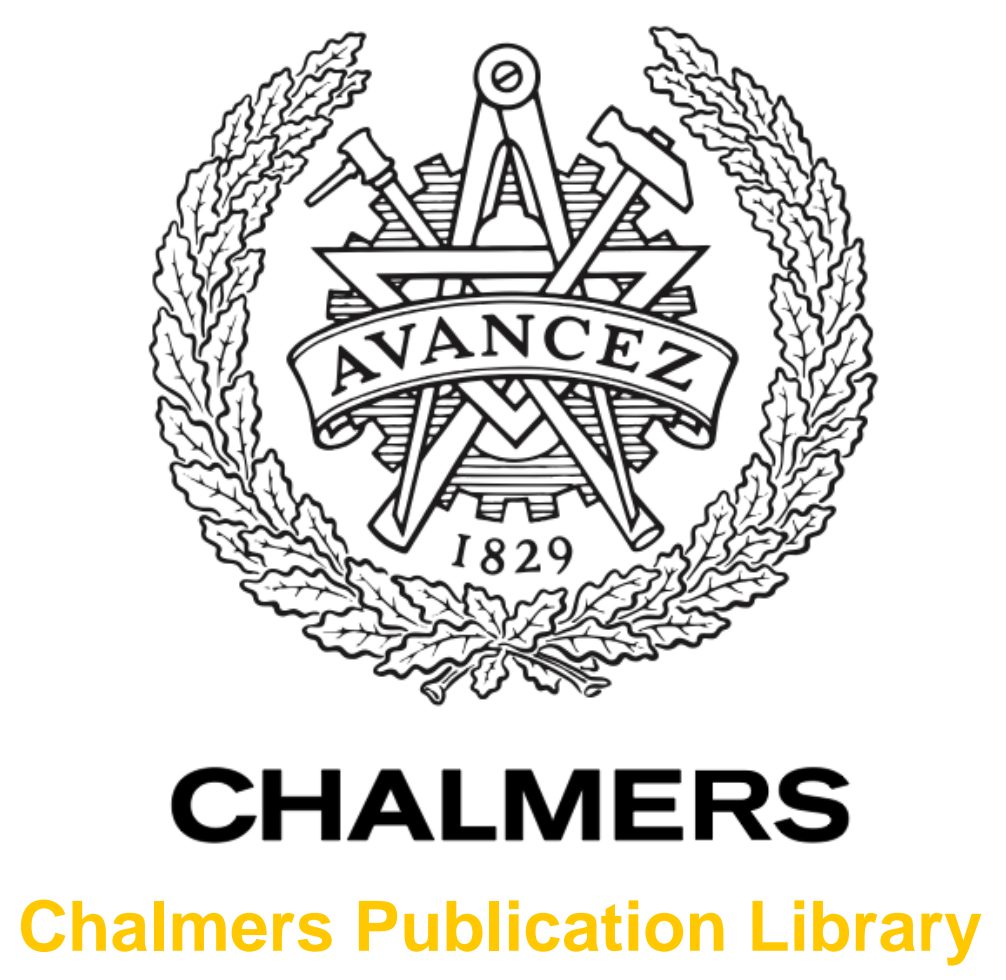

\title{
Nonlinear Impairment-Aware Static Resource Allocation in Elastic Optical Networks
}

This document has been downloaded from Chalmers Publication Library (CPL). It is the author's version of a work that was accepted for publication in:

Journal of Lightwave Technology (ISSN: 07338724)

Citation for the published paper:

Zhao, J. ; Wymeersch, H. ; Agrell, E. (2015) "Nonlinear Impairment-Aware Static Resource Allocation in Elastic Optical Networks". Journal of Lightwave Technology, vol. 33(22), pp. 4554-4564.

http://dx.doi.org/10.1109/JLT.2015.2474130

Downloaded from: http://publications.lib.chalmers.se/publication/228363

Notice: Changes introduced as a result of publishing processes such as copy-editing and formatting may not be reflected in this document. For a definitive version of this work, please refer to the published source. Please note that access to the published version might require a subscription. 


\title{
Nonlinear Impairment-Aware Static Resource Allocation in Elastic Optical Networks
}

\author{
Juzi Zhao, Henk Wymeersch, and Erik Agrell
}

\begin{abstract}
This paper studies the routing, modulation format, and spectrum allocation problem in elastic fiberoptical networks for static traffic. Elastic networks, based on Nyquist wavelength division multiplexing or optical orthogonal frequency division multiplexing, can efficiently utilize the optical fiber's bandwidth in an elastic manner by partitioning the bandwidth into hundreds or even thousands of subcarriers. Besides the amplified spontaneous emission noise, the nonlinear impairments of each connection is explicitly considered by utilizing an analytical model to calculate the nonlinear interference from other connections propagating in the same fibers. The objective of our work is to minimize the bandwidth, i.e., the number of used subcarriers, across the network, while satisfying demands on throughput and quality for all connections. A novel integer linear program formulation and low-complexity heuristics are proposed. Simulation results are presented to demonstrate the effectiveness of the proposed approaches. Compared with transmission reach-based benchmark methods, our methods can achieve up to $31 \%$ bandwidth reduction.
\end{abstract}

Index Terms-NWDM, OOFDM, elastic optical networks, routing and spectrum assignment, physical impairments.

\section{INTRODUCTION}

$\mathbf{N}$ ext generation backbone networks should have the capability to deal with the ever increasing and heterogeneous traffic from various applications, such as e-science and inter data center communications, which makes elastic optical networks an ideal candidate, since they can flexibly and efficiently utilize the fiber bandwidth. Nyquist wavelength division multiplexing (NWDM) and optical orthogonal frequency division multiplexing (OOFDM) are technologies to enable elastic optical networks. The optical spectrum of each fiber (e.g., the C-band) is divided into subcarriers. The bandwidth of subcarrier is much smaller (e.g., $6.25 \mathrm{GHz}$ or 12.5 GHz) than the bandwidth of the wavelengths in a fixed grid wavelength division multiplexing (WDM)-based optical network (e.g., $50 \mathrm{GHz}$ ). A variety of modulation schemes (e.g., quadrature phase-shift keying (QPSK) and 16-point quadrature amplitude modulation (16-QAM)) can be adopted by different subcarriers and result in different bit rates. Therefore, only the required number of subcarriers need to be allocated to a service in order to satisfy its throughput requirement [1].

There are three main challenges in the routing, modulation format, and spectrum allocation (RMSA) problem in elastic optical networks: (a) the spectrum continuity constraint, (b) the spectrum contiguity constraint, and (c) accounting for physical layer impairments. The spectrum

The authors are with the Department of Signals and Systems, Chalmers University of Technology, Gothenburg, Sweden (e-mail: juzi@chalmers.se, henkw@chalmers.se, and agrell@chalmers.se). The research was funded by the Swedish Research Council (VR) under grant no. 2012-5280.

This work was published in part at the Optical Fiber Communication Conference (OFC) March 2015. continuity constraint ensures that, when there are no spectrum converters in the network, the same set of subcarriers are assigned on all the links of a light-path. The spectrum contiguity constraint ensures that the subcarriers allocated to a connection are contiguous. Finally, physical layer impairments such as noise and crosstalk cause the quality of the optical signals to degrade as they traverse the network.

The connection requests for the RMSA problem can be static (offline) or dynamic (online). For static traffic, the connection requests in the form of a traffic matrix are given and fixed, rendering the RMSA problem into a planning problem. Once the planning state is completed, RMSA with dynamic traffic is considered during network operation, and connection requests arrive to and depart from the network following a stochastic process. In this paper, we focus exclusively on the static traffic problem.

\section{A. Related Work}

Most existing related work on impairment-aware RMSA in elastic optical networks are based on transmission-reach limits [5]-[10]. In these works, each modulation format has a corresponding transmission reach limit, which depends only on the linear impairments. The quality of a path assigned a particular modulation format is assumed to be good as long as the length of the path is no longer than the corresponding transmission reach. In addition, in order to avoid any nonlinear interference, a guard-band consisting of a number of subcarriers is inserted between two different connections that are assigned adjacent subcarrier bands.Nonlinear impairments were taken into account in [11] to derive worstcase transmission reach limits, assuming a full network load. In particular, the concept of distance-adaptive spectrum resource allocation was first proposed in [5]. This concept was applied to the RMSA problem for dynamic traffic in [6]-[8], and for static traffic in [9], using a novel integer linear programming (ILP) formulation and related heuristics. Finally, [10] considered the RMSA problem along with transponder allocation. However, the transmission-reach model fails to consider the actual nonlinear impairments (NLI), such as the interference among connections, which are dependent on the routing and resource allocation of connections over the same path. Hence, the reach and guard-band may overestimate or underestimate the connection impairments. More sophisticated methods that account for NLI were studied for dynamic and static routing and wavelength assignment $(\mathrm{RWA})^{1}$ problems in fixed-grid WDM networks (e.g., [12][17]). For the RMSA problem in elastic optical networks, effective methods were proposed for dynamic traffic in [11], for sequentially loaded traffic in [18], and for dynamic traffic

\footnotetext{
${ }^{1}$ The terminology of RWA is used in the WDM networks literature, where wavelengths are allocated to light-paths. In mixed line rate optical networks, modulation format allocation is part of RWA, since each line rate has a specific modulation format.
} 
TABLE I: Parameters for SNR calculation

\begin{tabular}{cl}
\hline Symbol & \multicolumn{1}{c}{ Meaning } \\
\hline$G$ & signal power spectral density \\
\hline$G_{\mathrm{ASE}}$ & PSD of ASE \\
\hline$G_{\mathrm{ASE}}^{0}$ & PSD of ASE of a single span \\
\hline$G_{\mathrm{NLI}}$ & PSD of NLI \\
\hline$G_{\mathrm{NLI}}^{l}$ & PSD of NLI of a single span on link $l$ \\
\hline$N_{l}$ & the number of spans (with one amplifier per span) \\
\hline$L$ & on link $l$ \\
\hline$\alpha$ & the length of each span \\
\hline$n_{s p}$ & the power attenuation \\
\hline $\mathrm{h}$ & the spontaneous emission factor \\
\hline$\nu$ & Planck's constant \\
\hline$j$ & the optical carrier frequency \\
\hline$B_{i}$ & another connection using link $l$ \\
\hline$B_{j}$ & the bandwidths for connections $i$ \\
\hline$\Delta f_{i j}$ & the center frequency spacing between connections $i$ \\
\hline$\gamma$ & and $j$ \\
\hline$\beta_{2}$ & the fiber nonlinearity coefficient \\
\hline$\rho$ & the fiber dispersion \\
\hline$\mu$ & $\left(\pi^{2}\left|\beta_{2}\right|\right) / \alpha$ \\
\hline
\end{tabular}

allocation in elastic optical networks with multi-core fibers in [19]. The problem of static RMSA in elastic optical networks under a realistic NLI model was studied for the first time in our conference paper [20]. In this paper, we include mathematically complete and self-contained descriptions of the ILP formulations and algorithms that were used in [20]. Furthermore, the results in [20] are extended by improving the proposed heuristics adding two more connection sorting policies in both group ILP (GILP) and connection list (CL), adding K-shortest paths and using simulated annealing in CL; and one more network topology is used in simulations.

\section{B. Contributions}

To the best of our knowledge, the static RMSA problem has not been addressed in elastic optical networks when taking into consideration both linear and nonlinear impairments. We provide an RMSA method based on a novel ILP formulation, as well as several low-complexity heuristics. Through extensive simulations, we show that our algorithms exhibit better performance compared to the algorithms considering transmission reach limits and guard-bands in [9].

This paper is organized as follows. In Section II, we present the physical layer impairment model and the problem statement. The ILP formulation, proposed heuristics and benchmark algorithms are presented in Section III. Section IV presents and discusses the numerical results. We conclude the paper and present possible extensions to this work in Section V.

\section{Physical Layer Impairment Model and Problem STATEMENT}

\section{A. Physical Layer Impairment Model}

Both amplified spontaneous emission noise (ASE) and NLI are considered by adopting the physical layer model proposed in [21]. Suppose connection $i$ is allocated route $r_{i}$, then its signal-to-noise-ratio ( $\mathrm{SNR})$ is

$$
\mathrm{SNR}_{i}=\frac{G}{G_{\mathrm{ASE}}+G_{\mathrm{NLI}}},
$$

where $G_{\mathrm{ASE}}=\sum_{l \in r_{i}} N_{l} G_{\mathrm{ASE}}^{0}$ and $G_{\mathrm{ASE}}^{0}=\left(e^{\alpha L}-1\right) n_{s p} \mathrm{~h} \nu$. Similarly, $G_{\mathrm{NLI}}=\sum_{l \in r_{i}} N_{l} G_{\mathrm{NLI}}^{l}$, and

$$
G_{\mathrm{NLI}}^{l}=\mu\left(\ln \left(\rho B_{i}^{2}\right)+\sum_{j} \ln \frac{\Delta f_{i j}+B_{j} / 2}{\Delta f_{i j}-B_{j} / 2}\right) .
$$

The related parameters are defined in Table I. (2) is only valid for bandwidths of $28 \mathrm{GHz}$ or more. The interested reader is referred to [21] for further details.

\section{B. Problem Statement}

The network consists of a set of nodes, which are connected by links. Each link consists of two fibers with opposite directions, on which a certain maximum bandwidth is available. This bandwidth is allocated by means of subcarrier modulation, realized as either OOFDM or NWDM. The spectrum is therefore allocated in multiples of the subcarrier bandwidth $C_{s}$. A set of connection requests between pairs of nodes should be simultaneously satisfied. For each connection in the network, a route, a modulation format, and a contiguous subcarrier band is allocated in order to satisfy its bit rate and SNR requirements. The goal is to minimize the maximum allocated subcarrier index on any link in the network, which corresponds to the maximum bandwidth. The set of static connection requests is represented by an all-to-all symmetric matrix. Each connection is bidirectional and the same resources are allocated in both directions.

\section{Proposed Algorithms And Benchmark}

To solve the RMSA problem, we first present an ILP formulation in Section III-A, that can deal with small problem instances. This approach is consistent with previous works, where small networks with 4-10 nodes were handled optimally using ILP, whereas various suboptimal heuristics were applied to larger networks with 14-28 nodes [9], [22], [23]. We then present two heuristics in Section III-B for larger instances. Finally, the benchmark ILP and heuristics are summarized in Section III-C.

\section{A. ILP Formulation}

The RMSA problem with the objective to minimize the maximum allocated subcarrier index on all links can be formulated as an ILP problem. The input parameters and variables used in the ILP are listed in Tables II and III, respectively. Thus, we can have the optimization problem as follows (as an extension of [9] by replacing the GN model based-SNR constraints with the reach-based constraints):

minimize $F$

s.t.

$\sum_{m} \mathcal{M}_{i m}^{\mathrm{mod}}=1 \quad \forall i$

$B_{i}=\sum_{m} T_{i m} \mathcal{M}_{i m}^{\bmod } \quad \forall i$

$F \geq f_{i}+B_{i}-1 \quad \forall i$

$\sum_{l} p_{i l}^{\text {link }} z_{l n}=2 p_{\text {in }}^{\text {node }}-v_{\text {in }} \quad \forall i, n$

$p_{i l}^{\text {link }}+p_{j l}^{\text {link }} \leq 1+y_{i j}^{\text {share }} \quad \forall i, j \neq i, l$

$f_{i}+B_{i}-f_{j} \leq \theta\left(1-u_{i j}^{\text {share }}\right) \quad \forall i, j \neq i$

$f_{j}+B_{j}-f_{i} \leq \theta\left(1-y_{i j}^{\text {share }}+u_{i j}^{\text {share }}\right) \quad \forall i, j \neq i$

$G \sum_{m} \mathcal{M}_{i m}^{\mathrm{mod}} / \mathrm{SNR}_{m} \geq G_{\mathrm{ASE}}^{0} \sum_{l} p_{i l}^{\text {link }} N_{l}+\sum_{l} N_{l} t_{i l}^{\mathrm{NLI}}$ 
TABLE II: ILP Input Parameters

\begin{tabular}{|c|c|}
\hline Symbol & Meaning \\
\hline$z_{l n} \in \mathbb{B}=\{0,1\}$ & $\begin{array}{l}z_{l n}=1 \text { if node } n \text { is an ending } \\
\text { node of link } l\end{array}$ \\
\hline$v_{\text {in }} \in \mathbb{B}$ & $\begin{array}{l}v_{i n}=1 \text { if } n \text { is source or desti- } \\
\text { nation of connection } i\end{array}$ \\
\hline$\Lambda_{i} \in \mathbb{R}_{\geq 0}$ & $\begin{array}{l}\text { bit rate requirement of connec- } \\
\text { tion } i\end{array}$ \\
\hline$T_{i m} \in \mathbb{N}$ & $\begin{array}{l}\text { number of subcarriers of con- } \\
\text { nection } i \text { when it is assigned } \\
\text { modulation format } m\end{array}$ \\
\hline $\mathrm{SNR}_{m} \in \mathbb{R}_{\geq 0}$ & $\begin{array}{l}\text { SNR threshold of modulation } \\
\text { format } m\end{array}$ \\
\hline$J_{\text {imh }}^{\text {crosstalk }}=\mu \ln \frac{h+T_{i m}}{h-T_{\text {im }}} \in \mathbb{R}_{\geq 0}$ & $\begin{array}{l}\text { the partial NLI introduced by } \\
\text { connection } i \text { to another connec- } \\
\text { tion when } i \text { is assigned modu- } \\
\text { lation format } m \text { and the cen- } \\
\text { ter frequency spacing between } \\
\text { them is } h C_{s} / 2, h \in \mathbb{N} \text { denotes } \\
\text { the number of subcarrier half } \\
\text { bandwidths }\end{array}$ \\
\hline$H_{i m}^{\mathrm{NLI}}=\mu \ln _{\mathbb{R}_{\geq 0}\left(\rho\left(C_{s} T_{i m}\right)^{2}\right) \in}$ & $\begin{array}{l}\text { the partial NLI of connection } i \\
\text { when it is assigned modulation } \\
\text { format } m\end{array}$ \\
\hline$N_{l} \in \mathbb{N}$ & number of spans on link $l$ \\
\hline$G \in \mathbb{R}_{\geq 0}$ & signal power spectrum density \\
\hline$G_{\mathrm{ASE}}^{0} \in \mathbb{R}_{\geq 0}$ & $\begin{array}{l}\text { ASE noise power spectrum } \\
\text { density per span }\end{array}$ \\
\hline$\theta \in \mathbb{N}$ & a large number \\
\hline
\end{tabular}

TABLE III: ILP Variables

\begin{tabular}{|c|c|}
\hline Symbol & Meaning \\
\hline$B_{i} \in \mathbb{N}$ & $\begin{array}{l}\text { the number of subcarriers allocated to connec- } \\
\text { tion } i\end{array}$ \\
\hline$f_{i} \in \mathbb{N}$ & $\begin{array}{l}\text { the lowest subcarrier index (first subcarrier } \\
\text { index) allocated to connection } i\end{array}$ \\
\hline$F \in \mathbb{N}$ & $\begin{array}{l}\text { the maximum allocated subcarrier index on } \\
\text { any link }\end{array}$ \\
\hline$p_{i l}^{\text {link }} \in \mathbb{B}$ & $\begin{array}{l}p_{i l}^{\text {link }}=1 \text { if link } l \text { is on the route assigned to } \\
\text { connection } i\end{array}$ \\
\hline$p_{\text {in }}^{\text {node }} \in \mathbb{B}$ & $\begin{array}{l}p_{i n}^{\text {node }}=1 \text { if node } n \text { is on the route assigned to } \\
\text { connection } i\end{array}$ \\
\hline $\mathcal{M}_{i m}^{\text {mod }} \in \mathbb{B}$ & $\begin{array}{l}\mathcal{M}_{\text {im }}^{\text {mod }}=1 \text { if connection } i \text { is assigned modula- } \\
\text { tion format } m\end{array}$ \\
\hline$y_{i j}^{\text {share }} \in \mathbb{B}$ & $\begin{array}{l}y_{i j}^{\text {share }}=1 \text { if connection } i \text { and connection } j \\
\text { share at least one common link }\end{array}$ \\
\hline$u_{i j}^{\text {share }} \in \mathbb{B}$ & $u_{i j}^{\text {share }}=1$ if $y_{i j}^{\text {share }}=1$ and $f_{i}+B_{i} \leq f_{j}$ \\
\hline$\Delta_{i j m}^{(h)} \in \mathbb{B}$ & $\begin{array}{l}\Delta_{i j m}^{(h)}=1 \text { if the center frequency spacing be- } \\
\text { tween connection } i \text { and connection } j \text { is } h C_{s} / 2 \\
(h \in \mathbb{N} \text { denotes the number of subcarrier half } \\
\text { bandwidths) and } \mathcal{M}_{j m}^{\text {mod }}=1\end{array}$ \\
\hline $\begin{array}{l}w_{i j l}^{\text {crosstalk }} \in \\
\quad \mathbb{R}_{\geq 0}\end{array}$ & $\begin{array}{l}\text { upper bound of the NLI to connection } i \text { from } \\
\text { connection } j \text { on link } l\end{array}$ \\
\hline$t_{i l}^{\mathrm{NLI}} \in \mathbb{R}_{\geq 0}$ & upper bound of $G_{\mathrm{NLI}}^{l}$ of connection $i$ on link $l$ \\
\hline
\end{tabular}

$t_{i l}^{\mathrm{NLI}} \geq \theta\left(p_{i l}^{\mathrm{link}}-1\right)+\sum_{m} \mathcal{M}_{i m}^{\mathrm{mod}} H_{i m}^{\mathrm{NLI}}+\sum_{j \neq i} w_{i j l}^{\text {crosstalk }} \quad \forall i, l$

$w_{i j l}^{\text {crosstalk }} \geq \theta\left(p_{j l}^{\text {link }}-1\right)+\sum_{m, h} \Delta_{i j m}^{(h)} J_{j m h}^{\text {crosstalk }} \quad \forall i, j \neq i, l$

$\sum_{h} \Delta_{i j m}^{(h)}=\mathcal{M}_{j m}^{\text {mod }} \quad \forall i, j \neq i, m$

$\sum_{m, h} h \Delta_{i j m}^{(h)} \leq \theta\left(1-u_{i j}^{\text {share }}\right)+2\left(f_{j}-1\right)+B_{j}$ $-2\left(f_{i}-1\right)-B_{i} \quad \forall i, j \neq i$

$\sum_{m, h} h \Delta_{i j m}^{(h)} \leq \theta\left(1-y_{i j}^{\text {share }}+u_{i j}^{\text {share }}\right)+2\left(f_{i}-1\right)+B_{i}$ $-2\left(f_{j}-1\right)-B_{j} \quad \forall i, j \neq i$.

The constraints are to be interpreted as follows: (3b) ensures that each connection is assigned only one modulation format; (3c) ensures that the number of subcarriers allocated to each connection depends on the modulation format assigned to this connection; (3d) ensures that the last allocated subcarrier index of each connection $i$ (i.e., $f_{i}+B_{i}-1$ ) does not exceed $F$; (3e) ensures that one no-loop route is allocated to each connection, ${ }^{2}$ (3f) ensures that $y_{i j}^{\text {share }}=1$ when connections $i$ and $j$ share at least one common link; $(3 \mathrm{~g})-(3 \mathrm{~h})$ ensure that when there is at least one common link shared by two connections $i$ and $j$, the spectrum allocated to them should not have any overlapping; ${ }^{3}$ (3i) ensures the satisfaction of the SNR requirement for connection $i$ considering the PSDs of ASE noise and NLI; (3j) determines the NLI PSD of connection $i$ on link $l ;^{4}(3 \mathrm{k})$ determines the NLI PSD from connection $j$ to connection $i$ on link $l 5^{5}$ (3l)-(3n) bound the value of $\Delta_{i j m}^{(h)}:(31)$ ensures that $\sum_{h} \Delta_{i j m}^{(h)}=1$ when connection $j$ is assigned modulation format $k$, i.e., $\mathcal{M}_{j m}^{\bmod }=1$; and $(3 \mathrm{~m})$ and $(3 \mathrm{n})$ ensure that $\sum_{m, h} h \Delta_{i j m}^{(h)}$ is no larger than ${ }^{6}$ the central frequency spacing between connections $i$ and $j$, accounting for the cases where connection $i$ is placed in a lower or a higher frequency band than connection $j$.

The number of variables and constraints in the ILP formulation depends on the number of connection requests $I$, the number of links $L$, the number of nodes $N$ in the network, the number of subcarriers on each link $S$, and the number of total modulation format $M$. In particular, $2 I+1$ integer variables, $2 I^{2}+I^{2} M S+I L+I N+I M$ boolean variables, and $I^{2} L+I L$ real variables. $I+I N+I^{2} M$ boolean equality constraints for (3b), (3e) and (3l); $I^{2} L$ boolean inequality constraints for (3f); I integer equality constraints for (3c); $I+4 I^{2}$ integer inequality constraints for $(3 \mathrm{~d}),(3 \mathrm{~g}),(3 \mathrm{~h}),(3 \mathrm{~m})$ and ( $3 \mathrm{n})$; and $I+I L+I^{2} L$ real inequality constraints for (3i), $(3 \mathrm{j})$ and $(3 \mathrm{k})$.

\section{B. Proposed Heuristics}

Although the routing, modulation format, and spectrum assignment of all connections can be optimally solved by the ILP, it is very time consuming due to the involved integer constraints, and it can only provide optimal results for small networks with a few connection requests within a reasonable time. Therefore, two heuristics, GILP and CL, are proposed for dealing with larger networks. In GILP, the requests are split into smaller groups and the requests within a group are optimally allocated using ILP, with the groups being sequentially processed. In CL, requests are allocated one by one.

${ }^{2}$ If node $n$ is on the route of connection $i$ (but not source or destination node of connection $i$ ), then two of the links ending with $n$ should be on the route, i.e., if $p_{i n}^{\text {node }}=1$ and $v_{i n}=0$, then $\sum_{l} p_{i l}^{\text {link }} z_{l n}=2$; if node $n$ is the source or destination node of connection $i$, then only one of the links ending with $n$ should be on the route, i.e., if $p_{i n}^{\text {node }}=1$ and $v_{i n}=1$, then $\sum_{l} p_{i l}^{\text {link }} z_{l n}=1$.

${ }^{3}$ Constraints of the form $a \leq \theta b+c$ are always true when $b>0$, by choice of a sufficiently large value of $\theta$.

${ }^{4}$ If link $l$ is not on the route of connection $i$, i.e., $p_{i l}^{\text {link }}=0$, then $(3 \mathrm{j})$ is inactive. If $p_{i l}^{\text {link }}=1$, then $t_{i l}^{\mathrm{NLI}} \geq \sum_{m} \mathcal{M}_{i m}^{\text {mod }} H_{i m}^{\mathrm{NLI}}+\sum_{j \neq i} w_{i j l}^{\text {crosstalk }}$, where the right side is the value of NLI of connection $i$ on link $l$.

${ }^{5}$ If link $l$ is not on the route of connection $j$, i.e., $p_{j l}^{\text {link }}=0$, then (3k) is inactive. On the other hand, if $p_{i l}^{\text {link }}=1$, then $w_{i j l}^{\text {crosstalk }} \geq$ $\sum_{m, h} \Delta_{i j m}^{(h)} J_{j m h}^{\text {crosstalk }}$, where the right side is the value of NLI to connection $i$ from connection $j$.

${ }^{6}$ According to (2), the nonlinear effect from connection $j$ to connection $i$ ( $w_{i j l}^{\text {crosstalk }}$ in the ILP formulation) increases as the central frequency spacing $\sum_{m, h} h \Delta_{i j m}^{(h)}$ decreases. Therefore, the ILP will set $\sum_{m, h} h \Delta_{i j m}^{(h)}$ to be equal to the actual central frequency spacing when necessary. 
TABLE IV: GILP Additional Variables

\begin{tabular}{|c|c|}
\hline Symbol & Meaning \\
\hline$f_{i e l}^{\prime} \in \mathbb{N}$ & $\begin{array}{l}\text { the lowest subcarrier index (first subcar- } \\
\text { rier index) allocated to connection } e \text { on } \\
\text { link } l \text { with respect to connection } i\end{array}$ \\
\hline$y_{i e l}^{\prime} \in \mathbb{B}$ & $\begin{array}{l}y_{i e l}^{\prime}=1 \text { if both connection } i \text { and connec- } \\
\text { tion } e \text { use link } l\end{array}$ \\
\hline$u_{i e l}^{\prime} \in \mathbb{B}$ & $u_{i e l}^{\prime}=1$ if $s_{i e l}^{\prime}=1$ and $f_{i}+B_{i} \leq f_{i e l}^{\prime}$ \\
\hline$\Delta_{i e l}^{\prime(h)} \in \mathbb{B}$ & $\begin{array}{l}\Delta_{i e l}^{\prime(h)}=1 \text { if on link } l, \Delta f_{i e}-B_{e} / 2= \\
C_{s} h / 2, \text { where } \Delta f_{i e} \text { is calculated based on } \\
f_{i e l}^{\prime}, h \in \mathbb{N} \text { denotes the number of subcar- } \\
\text { rier half bandwidths }\end{array}$ \\
\hline
\end{tabular}

When allocating one connection (or group of connections), the crosstalk from previously allocated connections will be considered. The algorithms also take the interference from $E \geq 1$ future connection requests (in GILP, these are the connections in next groups) into consideration by including an adaptive SNR margin. The interference from a future connection depends on its routing, modulation format, and central frequency, which are not known at this moment. To account for this, we make a conservative estimate: for the routing, we precompute $K$ shortest paths [24] for each connection $i$, and let $q_{i l}^{\prime}=1$ if any of the $K$ paths for connection $i$ uses link $l$. In terms of the modulation format, according to (2), the crosstalk to connection $i$ from connection $j$ depends on

$$
\ln \frac{\Delta f_{i j}+B_{j} / 2}{\Delta f_{i j}-B_{j} / 2}=\ln \left(1+\frac{B_{j}}{\Delta f_{i j}-B_{j} / 2}\right) .
$$

Therefore, the crosstalk achieves its maximum value when connection $j$ is assigned the lowest modulation format (i.e., largest bandwidth), and when $\Delta f_{i j}-B_{j} / 2$ takes its minimum possible value (note that it is not dependent on the bandwidth of connection $i$ ). We will assume these crosstalk values for the future $E$ connections.

Connection Ordering Policies: In both heuristics, the connections are first sorted by one of the three policies: ${ }^{7}$

(i) decreasing order of their bit rate requirements $\Lambda_{i}$;

(ii) decreasing order of number of links on their shortest paths;

(iii) decreasing order of $\Lambda_{i}$ times shortest path length.

The impact of these orderings will be evaluated in the numerical results. We now present the two heuristics in detail.

1) Group ILP (GILP): After ordering according to one of the three policies, GILP divides the connections into groups of size $\eta \geq 1$ (except the last group, whose size may be less than $\eta$ ). Each group is denoted as $\Theta_{a}$ for $1 \leq a \leq\lceil I / \eta\rceil$, where $I$ is the total number of connection requests. Then, a modification of ILP in (3) is called for each group.

For a particular group $a$, denote the set of already allocated connections (i.e., the connections that belong to previous groups) as $\Gamma=\cup_{a^{\prime}=1}^{a-1} \Theta_{a^{\prime}}$, and the set of $E$ future connections as $\Psi$. Besides the variables in Table III, additional variables are needed as shown in Table IV. This notation now allows us to formulate the following optimization problem for group

\footnotetext{
${ }^{7} \mathrm{We}$ also tried the sorting policy that the connections are sorted in decreasing order of their shortest path length, but its performance is dominated by other policies in all simulated cases in this paper.
}

$a$.

minimize

$\left\{\begin{array}{l}\theta F+\sum_{i \in \Theta_{a}, e \in \Psi, l, h} h \Delta_{i e l}^{\prime(h)}, \text { if } a=\lceil I / \eta\rceil \\ \theta F+\sum_{i \in \Theta_{a}}\left(f_{i}+B_{i}+\sum_{m} m \mathcal{M}_{i m}^{\text {mod }}\right)+\sum_{i \in \Theta_{a}, e \in \Psi, l, h} h \Delta_{i e l}^{\prime(h)} \\ \quad \text { otherwise }\end{array}\right.$

s.t.

$\sum_{m} \mathcal{M}_{i m}^{\text {mod }}=1 \quad \forall i \in \Theta_{a}$

$B_{i}=\sum_{m} T_{i m} \mathcal{M}_{i m}^{\text {mod }} \quad \forall i \in \Theta_{a}$

$F \geq f_{i}+B_{i}-1 \quad \forall i \in \Theta_{a}$

$\sum_{l} p_{i l}^{\text {link }} z_{l n}=2 p_{i n}^{\text {node }}-v_{i n} \quad \forall i \in \Theta_{a}, n$

$p_{i l}^{\text {link }}+p_{j l}^{\text {link }} \leq 1+y_{i j}^{\text {share }} \quad \forall i \in \Gamma \cup \Theta_{a}, j \in \Gamma \cup \Theta_{a} \backslash i, l$

$f_{i}+B_{i}-f_{j} \leq \theta\left(1-u_{i j}^{\text {share }}\right) \quad \forall i \in \Theta_{a}, j \in \Gamma \cup \Theta_{a} \backslash i$

$f_{j}+B_{j}-f_{i} \leq \theta\left(1-y_{i j}^{\text {share }}+u_{i j}^{\text {share }}\right) \quad \forall i \in \Theta_{a}, j \in \Gamma \cup \Theta_{a} \backslash i$

$G \sum_{m} \mathcal{M}_{i m}^{\text {mod }} / \mathrm{SNR}_{m} \geq G_{\mathrm{ASE}}^{0} \sum_{l}\left(p_{i l}^{\text {link }} N_{l}\right)+N_{l} \sum_{l} t_{i l}^{\mathrm{NLI}}, \forall i \in \Theta_{a}$

$t_{i l}^{\mathrm{NLI}} \geq \theta\left(p_{i l}^{\text {link }}-1\right)+\sum_{m} \mathcal{M}_{i m}^{\mathrm{mod}} H_{i m}^{\mathrm{NLI}}+\sum_{j \in \Gamma \cup \Psi \cup \Theta_{a} \backslash i} w_{i j l}^{\text {crosstalk }}$,
$\forall i \in \Theta_{a}, l$

$w_{i j l}^{\text {crosstalk }} \geq \theta\left(p_{j l}^{\text {link }}-1\right)+\sum_{m, h} \Delta_{i j m}^{(h)} J_{j m h}^{\text {crosstalk }}$

$$
\forall i \in \Theta_{a} \cup \Gamma, j \in \Gamma \cup \Theta_{a} \backslash i, l
$$

$\sum_{h} \Delta_{i j m}^{(h)}=\mathcal{M}_{j m}^{\bmod } \quad \forall i \in \Theta_{a} \cup \Gamma, j \in \Gamma \cup \Theta_{a} \backslash i, m$

$\sum_{m, h} h \Delta_{i j m}^{(h)} \leq \theta\left(1-u_{i j}^{\text {share }}\right)+2\left(f_{j}-1\right)+B_{j}$

$-2\left(f_{i}-1\right)-B_{i} \quad \forall i \in \Theta_{a} \cup \Gamma, j \in \Gamma \cup \Theta_{a} \backslash i$

$\sum_{m, h} h \Delta_{i j m}^{(h)} \leq \theta\left(1-y_{i j}^{\text {share }}+u_{i j}^{\text {share }}\right)+2\left(f_{i}-1\right)+B_{i}$ $-2\left(f_{j}-1\right)-B_{j} \quad \forall i \in \Theta_{a} \cup \Gamma, j \in \Gamma \cup \Theta_{a} \backslash i$

$p_{i l}^{\text {link }}+q_{e l}^{\prime} \leq 1+y_{i e l}^{\prime} \quad \forall i \in \Theta_{a} \cup \Gamma, e \in \Psi, l$

$f_{i}+B_{i}-f_{i e l}^{\prime} \leq \theta\left(1-u_{i e l}^{\prime}\right) \quad \forall i \in \Theta_{a} \cup \Gamma, e \in \Psi, l$

$f_{i e l}^{\prime}+T_{e M}-f_{i} \leq \theta\left(1-y_{i e l}^{\prime}+u_{i e l}^{\prime}\right), \forall i \in \Theta_{a} \cup \Gamma, e \in \Psi, l \quad(5 q)$

$\varphi_{i} \geq N_{l} \sum_{j \in \Theta_{a}} w_{i j l}^{\text {crosstalk }} \quad \forall i \in \Gamma$

$w_{i e l}^{\text {crosstalk }}=q_{e l}^{\prime} \sum_{h} \Delta_{i e l}^{\prime(h)} X_{e h} \quad \forall i \in \Theta_{a}, e \in \Psi, l$

$\sum_{h} h \Delta_{i e l}^{\prime(h)} \leq \theta\left(1-u_{i e l}^{\prime}\right)+2\left(f_{i e l}^{\prime}-1\right)-2\left(f_{i}-1\right)-B_{i}$

$\forall i \in \Theta_{a}, e \in \Psi, l$

$$
\begin{aligned}
\sum_{h} h \Delta_{i e l}^{\prime(h)} & \leq \theta\left(1-y_{i e l}^{\prime}+u_{i e l}^{\prime}\right)+2\left(f_{i}-1\right)+B_{i} \\
& -2\left(f_{i e l}^{\prime}-1\right)-2 T_{e M} \quad \forall i \in \Theta_{a}, e \in \Psi, l .
\end{aligned}
$$

Constraints (5b)-(5n) are the equivalents of (3b)-(3n), except that the values of $p_{i l}^{\text {link }}, p_{i n}^{\text {node }}, \mathcal{M}_{i m}^{\text {mod }}, f_{i}$, and $B_{i}$ of each connection $i \in \Gamma$ are known inputs, instead of variables. Constraints (5o)- $(5 \mathrm{u})$ are included to protect against interference from future connection requests, in which we introduce the following notation: in (5r), $\varphi_{i} \in \mathbb{R}_{\geq 0}$ denotes the remaining SNR of connection $i \in \Gamma$, which is calculated as $\varphi_{i}=G / \mathrm{SNR}_{\text {req }}-G_{\mathrm{ASE}}-G_{\mathrm{NLI}}^{\prime}$, where $\mathrm{SNR}_{\text {req }}$ is the required $\mathrm{SNR}$, according to the modulation scheme assigned to connection $i$, to achieve a certain threshold BER value, minus an adaptive SNR margin to account for a number $E$ of future connections, $G_{\mathrm{ASE}}$ is the PSD of ASE for connection $i$ (depending on its route), and $G_{\mathrm{NLI}}^{\prime}$ is the PSD of the NLI according to the resource allocation to connections in $\Gamma \cup \Psi$. Note that $\varphi_{i}$ is an input to the ILP in (5); $T_{e m}$ is the number of subcarriers requirement for connection $e$ when allocated modulation format $m$ and $X_{e h}=\mu \ln \left(1+2 T_{e 1} / h\right)$. Hence $T_{e M}$ 
is the smallest bandwidth requirement for connection $e$ and $X_{e h}$ is the largest crosstalk to connection $i$ from connection $e$.

The number of variables and constraints in the GILP formulation depends on the number of connection requests in each group $\eta$, the number of future connection requests $E$, the number of links $L$, the number of nodes $N$ in the network, the number of subcarriers on each link $S$, and the number of total modulation format $M$. In particular, $2 \eta+1+\eta E L$ integer variables, $2 \eta^{2}+\eta^{2} M S+\eta L+\eta N+$ $\eta M+2 \eta E L+\eta E S L$ boolean variables, and $\eta^{2} L+\eta L$ real variables. $\eta+\eta N+2 \eta I M$ boolean equality constraints for (5b), (5e) and (5l); $2 \eta I L+I E L$ boolean inequality constraints for (5f) and (5o); $\eta$ integer equality constraints for (5c); $\eta+2 \eta I L+2 \eta I+2 I E L+2 \eta E L$ integer inequality constraints for $(5 \mathrm{~d}),(5 \mathrm{~g}),(5 \mathrm{~h}),(5 \mathrm{~m}),(5 \mathrm{n}),(5 \mathrm{p}),(5 \mathrm{q}),(5 \mathrm{t})$ and $(5 \mathrm{u}) ; \eta E L$ real equality constraints for (5s); and $\eta+\eta L+2 \eta I L+I$ real inequality constraints for (5i), (5j), (5k) and (5r).

After solving (5) for the group $\Theta_{a}$, the values of $\varphi_{i}, i \in \Gamma$ are updated (by adding the nonlinear impairments from connections $\in \Theta_{a}$ to $G_{\mathrm{NLI}}^{\prime}$ ) and $\varphi_{i}, i \in \Theta_{a}$ are computed (by calculating the value of $G / \mathrm{SNR}_{\text {req }}-G_{\mathrm{ASE}}-G_{\mathrm{NLI}}^{\prime}$, where $G_{\mathrm{NLI}}^{\prime}$ includes the NLI PSD from connections in $\Theta_{a} \cup \Gamma \cup \Psi$ ). In case (5) is not feasible, this means that the choice of $E$ was too aggressive and must be increased. All requests are then reprocessed with a larger value of $E$, thus accounting for more nonlinear crosstalk from future connections. As $E$ increases, the $\mathrm{SNR}_{\text {req }}$ for previous allocated connections decreases, therefore, there may be feasible solutions for current group without exceeding the $\mathrm{SNR}_{\text {req }}$ (with increased $E$ ) of previous allocated groups. All together, this leads to the GILP method, summarized in Algorithm 1.

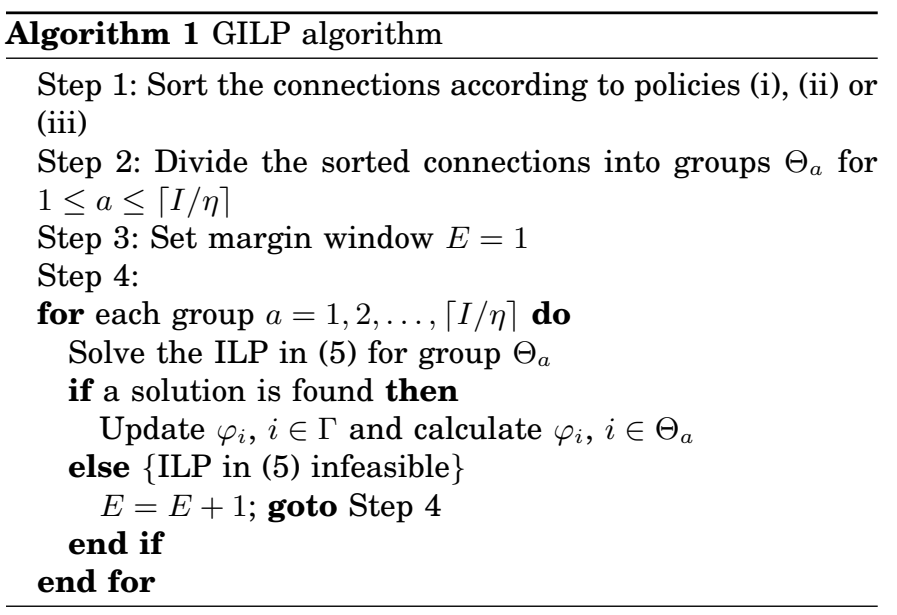

2) Connection List (CL): In CL, we allocate the connections one by one. For each connection, we utilize the $K$ minimum cost paths algorithm [24]. Connections are assumed to be ordered according to a certain policy.

Before formulating the CL algorithm, we introduce the following new notation: $f_{i}$ denotes the first subcarrier index allocated to connection $i ; \omega_{i}$ denotes the last subcarrier index allocated to connection $i ; \sigma_{s l} \in \mathbb{B}$ denotes the state of subcarrier $s$ on link $l$, with $\sigma_{s l}=1$ if the subcarrier $s$ on link $l$ is available; $p_{i l}^{\text {link }}=1$ if link $l$ is on the route $r_{i}$ of connection $i$; and $m_{i}$ denotes the modulation level allocated to connection $i$. For connection $i$, given a certain trial modulation format $m^{\prime}$ and a certain first subcarrier index $f^{\prime}$, we associate a cost $c_{l}$ with each link $l$, defined as

$$
c_{l}= \begin{cases}+\infty, & \text { if } \exists s \in\left[f^{\prime}, f^{\prime}+T_{i m^{\prime}}-1\right]: \sigma_{s l}=0 \\ N_{l} G_{\mathrm{ASE}}^{0}+N_{l} \mu \ln \left(\rho T_{i m^{\prime}}^{2}\right)+c_{l}^{\text {past }}+c_{l}^{\text {fut }} \text { otherwise }\end{cases}
$$

in which $c_{l}^{\text {past }}=N_{l} \mu \sum_{j=1}^{i-1} p_{j l}^{\text {link }} \ln \left[\left(\Delta f_{i j}+B_{j} / 2\right) /\left(\Delta f_{i j}-B_{j} / 2\right)\right]$ is the partial NLI from each previously allocated connection $j$ whose route includes link $l$, and $c_{l}^{\text {fut }}=N_{l} \sum_{e=i+1}^{i+E} q_{e l}^{\prime} X_{e h^{\prime}}^{\prime}$ is the estimated NLI from $E$ future connections. Here $X_{e h^{\prime}}^{\prime}=$ $\mu \ln \left[1+T_{e 1} /\left(h^{\prime}-T_{e M} / 2\right)\right]$ and $h^{\prime}$ is the smallest possible central frequency spacing between the connection $i$ and $e$ with the assumption that the modulation format allocated to connection $i$ is $m^{\prime}$, the first subcarrier index allocated to connection $i$ is $f^{\prime}$, and the modulation format for connection $e$ is $M$ (the highest one). Note that $c_{l}=\infty$ if any subcarrier $f^{\prime} \leq s \leq f^{\prime}+T_{i m^{\prime}}-1$ is not available.

The CL method now operates as follows: we initialize $E=1, \omega_{i}=+\infty$ for every connection $i$, and $\sigma_{s l}=1$ for all $s, l$. Then, for each connection $i$, ordered according to a given policy, every possible modulation format $1 \leq m^{\prime} \leq M$, and every possible first subcarrier index $f^{\prime}$ will be tried. For each $\left(m^{\prime}, f^{\prime}\right)$ candidate, $K^{\prime}$ minimum cost paths will be determined [24], utilizing the link costs in (6).

For each of the $K^{\prime}$ candidate paths for connection $i$ (with modulation format candidate $m^{\prime}$ and first subcarrier index candidate $f^{\prime}$ ), we check two conditions: (C-1) the SNR requirement of connection $i$ should be satisfied, i.e., $\sum_{l \in \text { path }} c_{l} \leq G / \mathrm{SNR}_{m^{\prime}}$, and (C-2) the SNR requirement of each previously allocated connection $j<i$ is not violated after allocating connection $i$ with the candidate path, modulation formation $\mathrm{m}^{\prime}$, and first subcarrier $f^{\prime}$. Finally, the route, modulation format, and first subcarrier index for connection $i$ will be selected as the one that achieves the smallest $\omega_{i}$. If there is no feasible allocation for connection $i$, then we set $E=E+1$, and we repeat the whole allocation procedure for all connections. The algorithm is summarized in Algorithm 2.

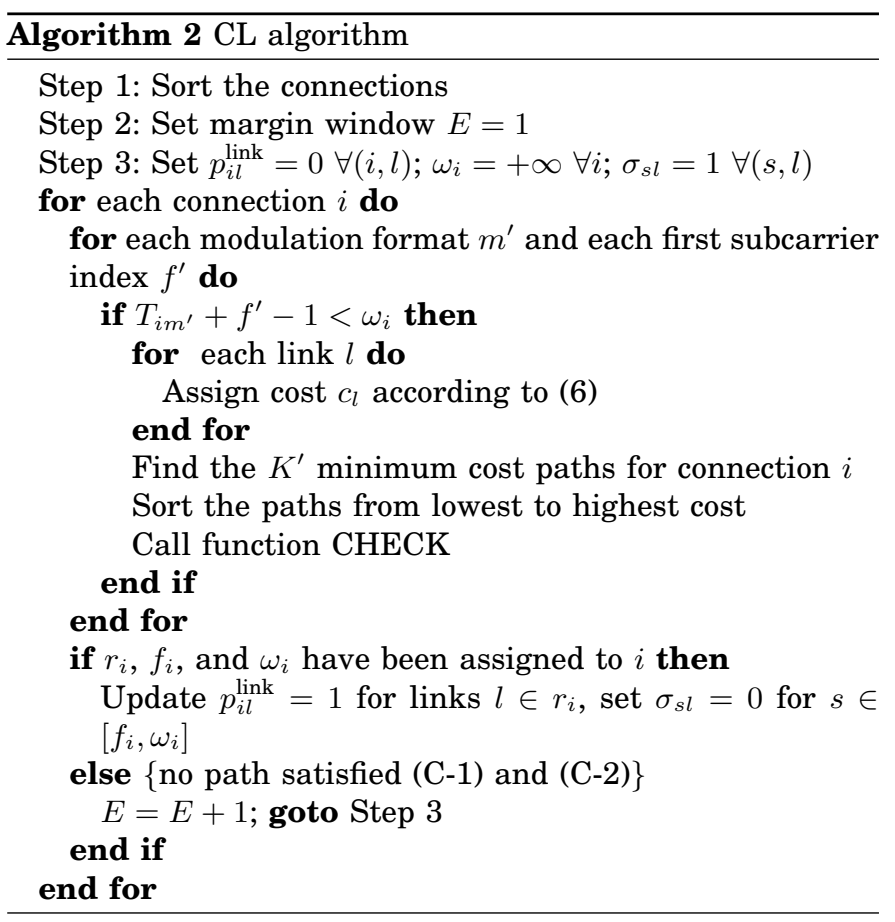




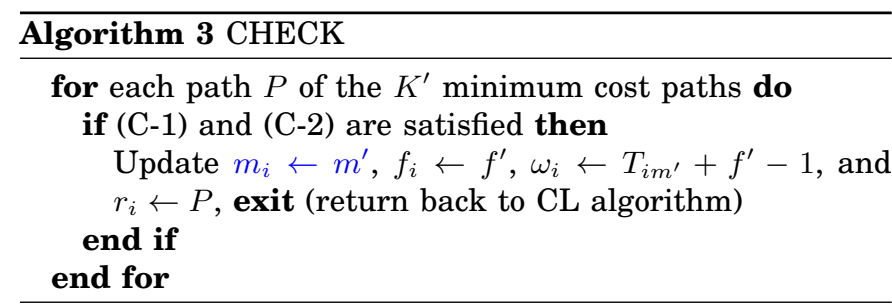

We utilize simulated annealing to improve the CL heuristic performance (CL-SimAn). Simulated annealing is performed by the Matlab built-in simulated annealing heuristic with default reannealing policy, temperature function, and acceptance function (i.e., the function used to determine whether a new point is accepted or not). For the required custom annealing function, a trial point for the problem is an ordering of connections. The custom annealing function for the problem will take the connection order with connection ordering policy (i) as input. The annealing function then modifies this ordering and returns a new ordering (i.e., a new point) by interchanging a uniformly selected connection with another uniformly selected connection. An objective function for the problem is another required input. The objective function returns the maximum allocated subcarrier index on any link for a given ordering. The proposed CL heuristic, without the connection sorting step (i.e., Step 1 in Algorithm 2 is removed) is used as the objective function. The SimAn algorithm stops if the number of iterations exceeds a maximum number of iterations, which is set to 10000 in all SimAn simulations.

\section{Benchmarks}

To compare the performance of our proposed ILP, GILP, and CL methods, we utilize the ILP and heuristics proposed in [9] as benchmarks. The benchmark relies on a combination of transmission reach limits and guard-band, in order to deal with physical layer impairments. In particular, there is a transmission reach limit $R_{m}$ for each modulation format $m$, so that a path longer than $R_{m}$ cannot be assigned modulation format $m$. A guard-band of $g$ subcarriers is used to avoid the interference between connections, instead of estimating the actual NLI. For each pair of nodes, $K=3$ paths are precomputed based on a proposed modified shortest path algorithm [9]. The route for each connection is then selected from the corresponding $K$ paths. These algorithms are summarized here, while the interested reader is referred to [9] for further details.

1) ILP: An ILP formulation for the RMSA problem based on transmission reach limit was presented in [9].

2) $R M L+S A I L P$ : The RMSA problem is divided into two subproblems: the routing and modulation level assignment (RML) problem, and the spectrum assignment (SA) problem. The subproblems are solved sequentially. In other words, the SA problem uses the output of RML problem. The ILP formulation in [9] is applied to each subproblem. For large network, since it takes a lot of time to obtain the optimal solution for SA problem, its ILP program is aborted after a fixed period of time, so the recorded results might not be optimal.

3) RMSA heuristic: The RMSA heuristic allocates the connections one by one. The connections are sorted by (i) decreasing order of their bit rate requirements $\Lambda_{i}$; or (ii) decreasing order of the number of links on their shortest paths. For each of the $K$ paths of every pair of nodes, the modulation format of the path is assigned as the highestorder modulation format whose reach limit $R_{m}$ is longer than or equal to the length of the path. For each connection, the algorithm calculates the spectrum availability of all the candidate $K$ paths based on the spectrum availability of links on the paths. The algorithm selects the path whose first available and sufficient subcarrier-band (i.e., the subcarrierband is wide enough for the bandwidth requirement $T_{i m}$ of the current connection $i$ ) has the lowest first subcarrier index. In addition to the two connection sorting policies, a simulated annealing (SimAn) method was used to improve the performance of the RMSA heuristic by finding good ordering of the connections. There is a fixed transmission reach limit for each modulation format with a particular signal power in [9]. In order to deal with different values of power spectral density $G$ in this work, we set the reach for modulation format $k$ as $\left\lfloor G /\left(\mathrm{SNR}_{m} G_{\mathrm{ASE}}^{0}\right)\right\rfloor$ spans.

\section{NUMERICAL RESULTS}

\section{A. Simulation settings}

There are two network topologies for simulations: a small 6-node network (Fig. 1) and the larger 14-node Deutsche Telekom (DT) network (Fig. 2). The number on each link corresponds to the number of spans. The parameters related to physical impairments are $\alpha=0.22 \mathrm{~dB} / \mathrm{km}, \gamma=1.32$ $(\mathrm{W} \mathrm{km})^{-1}, \beta_{2}=-21.7 \mathrm{ps}^{2} / \mathrm{km}, n_{s p}=1.8, \nu=193 \mathrm{THz}$, $L=100 \mathrm{~km}$, and $C_{s}=12.5 \mathrm{GHz}$ [25], [26]. The bit error rate (BER) requirement for all connections is set to $10^{-3}$. There are $M=4$ modulation formats: BPSK, QPSK, 8-QAM, and 16-QAM. We consider two kinds of bit rate requirements: the low bit rate requirement of each connection is uniformly distributed from $312.5 \mathrm{Gbps}$ to $625 \mathrm{Gbps}$; the high bit rate requirement of each connection is uniformly distributed from 1250 Gbps to $3750 \mathrm{Gbps}^{8} \mathrm{~K}$ is set to be 3 for the CL, GILP heuristics and benchmark algorithms, and $K^{\prime}=5$ for the CL heuristic. The guardband used for the benchmark methods is set to $g=1$ and $g=2$ subcarriers. For each simulation, the maximum allocated subcarrier index (on any link in the topologies) is recorded. Ten connection request matrices are generated, and the average results are shown in the following results figures.

\section{B. Results for 6-node network}

For the small 6-node network, the results for the proposed ILP, proposed heuristics (CL and GILP) with three connection sorting policies (CL-(i), CL-(ii), CL-(iii), GILP-(i),

${ }^{8}$ The lowest bit requirement ensures that even with the $16 \mathrm{QAM}$ modulation format, the number of allocated subcarriers is more than three, which is larger than the $28 \mathrm{GHz}$ bandwidth limit with the physical layer model (see Sec. II-A).

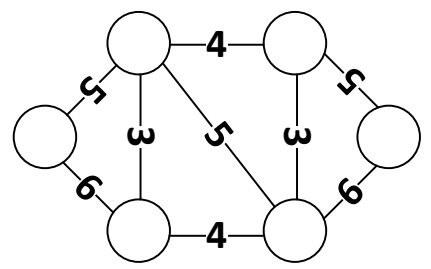

Fig. 1: 6-node network. The number on each link corresponds to the number of spans. 
GILP-(ii), and GILP-(iii), respectively), CL-SimAn, and the benchmark ILP are compared. We run the simulations on a desktop computer with 4-core, i7-2600 CPU @3.40GHz, and 8 GB RAM. The typical running time for ILP is 3 hours. The GILP (with group size $\eta=1$ ) for one $E$ value is 10 minutes, but it may need to run multiple $E$ values to get a feasible solution.

When the bit rate requirement of each connection is between $312.5 \mathrm{Gbps}$ and $625 \mathrm{Gbps}$, the resulting average maximum bandwidths as a function of the PSD ( $G$ in (2)) for the small network is shown in Fig. 3. As shown in Fig. 3(a), at higher PSD values, the guardband with one and two subcarriers in the benchmark ILP is insufficient to account for the NLI, rendering the optimization infeasible. SimAn and GILP-(i) with group size $\eta=1$ have better performance than CL-(i). The optimal ILP saves $15 \%$ bandwidth over CLSimAn when $G=0.02 \mathrm{~W} / \mathrm{THz}$. As shown in Fig. 3(b), since the shortest path distances and the bandwidth requirements for the connections are similar to each other, the sorting policies do not affect the results much. CL-SimAn has better results than all CL algorithms with different policies (i)-(iii). Also, the results of GILP with group size $\eta=1$ are better than the CL algorithms.

After scaling the network topology by doubling each link length as in [10], we get the results in Fig. 4. In this case, the guardband with one subcarrier in the benchmark ILP fails to produce feasible solutions; compared with the network with the original link length, the NLI is increased, since it is proportional to the link length according to (2).The optimal ILP can save up to $22 \%$ subcarriers compared with the benchmark ILP of two subcarrier guardband when $G=0.015$ $\mathrm{W} / \mathrm{THz}$.

When the bit rate requirement of each connection increases to the range between $1250 \mathrm{Gbps}$ and $3750 \mathrm{Gbps}$, the resulting bandwidths as a function of the PSD ( $G$ in Table I) are shown in Fig. 5. The results confirm that when the value of PSD is high, the benchmark fails to provide feasible solutions. The ILP provides up to $19 \%$ bandwidth reduction (compared with the benchmark ILP with $g=2$ ) when $G=0.015 \mathrm{~W} / \mathrm{THz}$. After scaling the network topology by doubling each link length, we get the results in Fig. 6 .

Besides uniform traffic, we also consider a normalized traffic demand matrix used in [27]. The normalized traffic demand $\Lambda_{s, d}^{\prime}$ for each pair of nodes $s, d$ is calculated as (for $s \neq d), \Lambda_{s, d}^{\prime} \propto \exp \left(Z_{s d} / Z^{0}\right)$, where $Z^{0}$ is a characteristic traffic distance and $Z_{s, d}$ is the length of shortest path between node $s$ and node $d$. Demands are normalized by

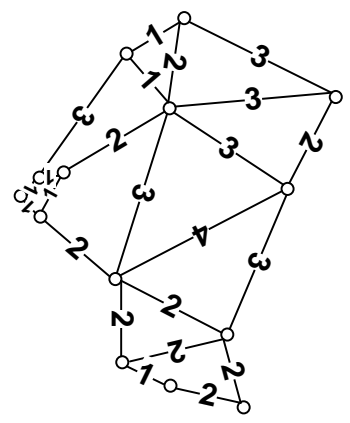

Fig. 2: 14-node DT network. The number on each link corresponds to the number of spans.

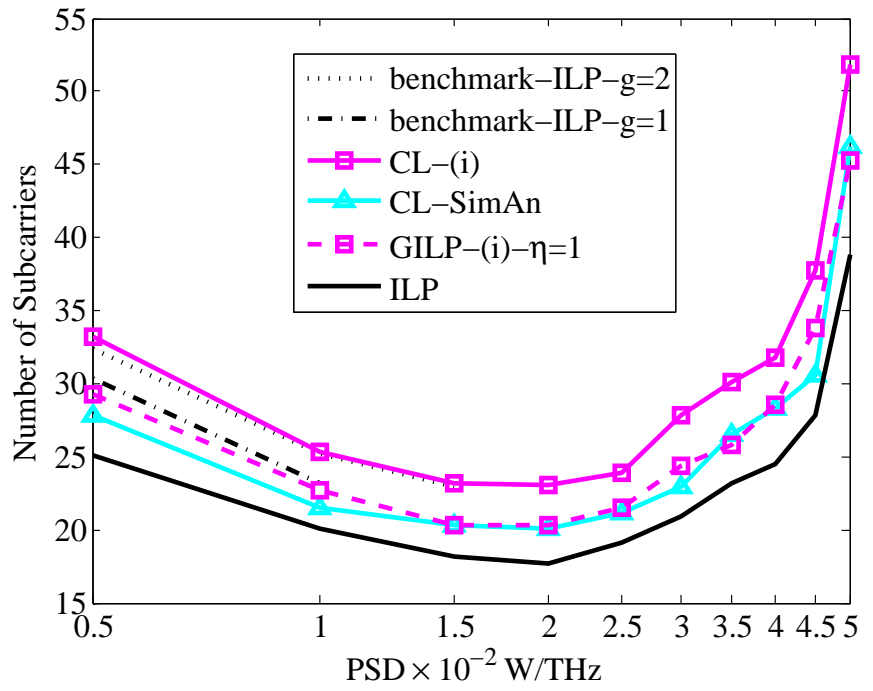

(a)

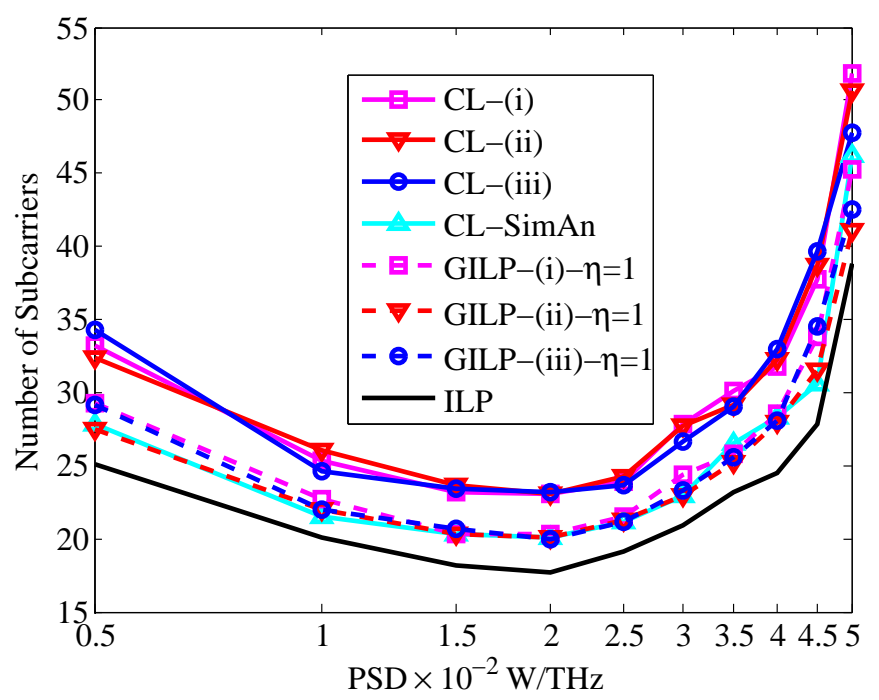

(b)

Fig. 3: The bandwidth (average of maximum allocated subcarrier indexes) vs. PSD ( $G$ in Table I) for the bit rate requirement of each connection is between $312.5 \mathrm{Gbps}$ and 625 Gbps in the 6-node network. (a) Main algorithms compared with benchmarks. (b) Proposed algorithms, including the sorting policies.

$\sum_{s, d} \Lambda_{s, d}^{\prime}=1$, and the actual traffic demand $\Lambda_{s, d}=Q \Lambda_{s, d}^{\prime}$, where $Q=5000, Z^{0}=1000 \mathrm{~km}$ are used in the simulation. The results are shown in Fig. 7. Again, when the value of PSD is high, the benchmark fails to provide feasible solutions. Furthermore, The ILP provides $13 \%$ bandwidth reduction (compared with the benchmark ILP with $g=2$ ) when $G=0.015 \mathrm{~W} / \mathrm{THz}$.

\section{Results for 14-node network}

The ILP and GILP are too time consuming to provide optimal results for the larger 14-node network, ${ }^{9}$ so we only

${ }^{9}$ The reason why GILP is time consuming is that for each value of $E$, if there is no feasible solution, then the heuristic will run again from the beginning, which results in many re-running of the GILP heuristic. 


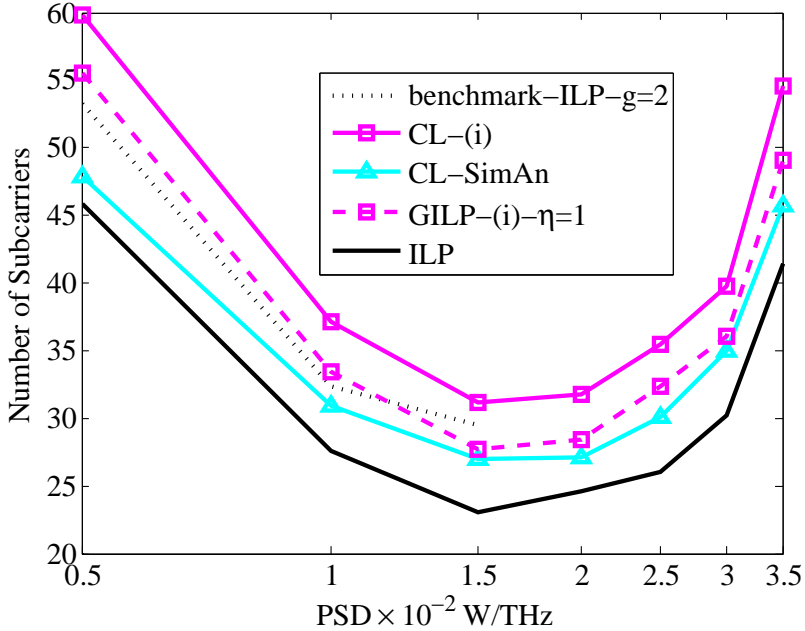

(a)

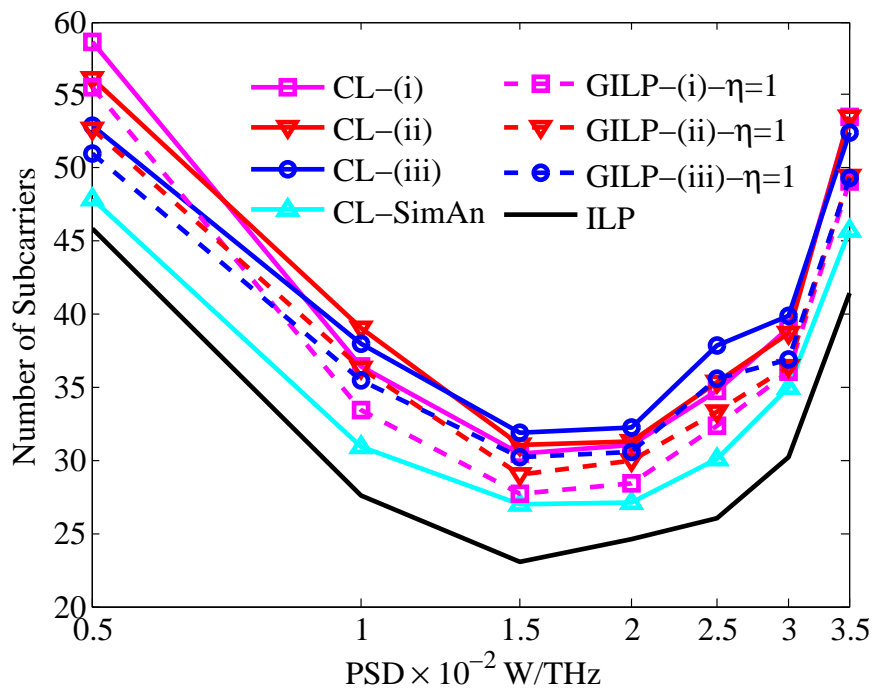

(b)

Fig. 4: The bandwidth (average of maximum allocated subcarrier indexes) vs. PSD ( $G$ in Table I) for the bit rate requirement of each connection is between 312.5 Gbps and $625 \mathrm{Gbps}$ in the 6-node network with doubled link length. (a) Main algorithms compared with benchmarks. (b) Proposed algorithms, including the sorting policies.

present results of the proposed CL and benchmark heuristics RML+SA ILP (aborted after 4-hour simulation), the RMSA heuristic with two connection sorting policies (RMSA-(i) and RMSA-(ii)), and RMSA-SimAn.

In the case where the bit rate requirement of each connection is between $312.5 \mathrm{Gbps}$ and $625 \mathrm{Gbps}$, the results are shown in Fig. 8. Compared with RMSA-SimAn with $g=2$, CL-SimAn can save up to $31 \%$ bandwidth when $G=0.015 \mathrm{~W} / \mathrm{THz}$. Also, the policies (ii) and (iii) have better performance than policy (i), which shows that the path distance is a more important factor than the number of required subcarriers in this case.

In the case where the bit rate requirement of each connection is between $1250 \mathrm{Gbps}$ and $3750 \mathrm{Gbps}$, the results are shown in Fig. 9. The results confirm that the benchmark heuristics provide feasible solutions only when PSD is low. CL-SimAn achieves up to $22 \%$ bandwidth reduction

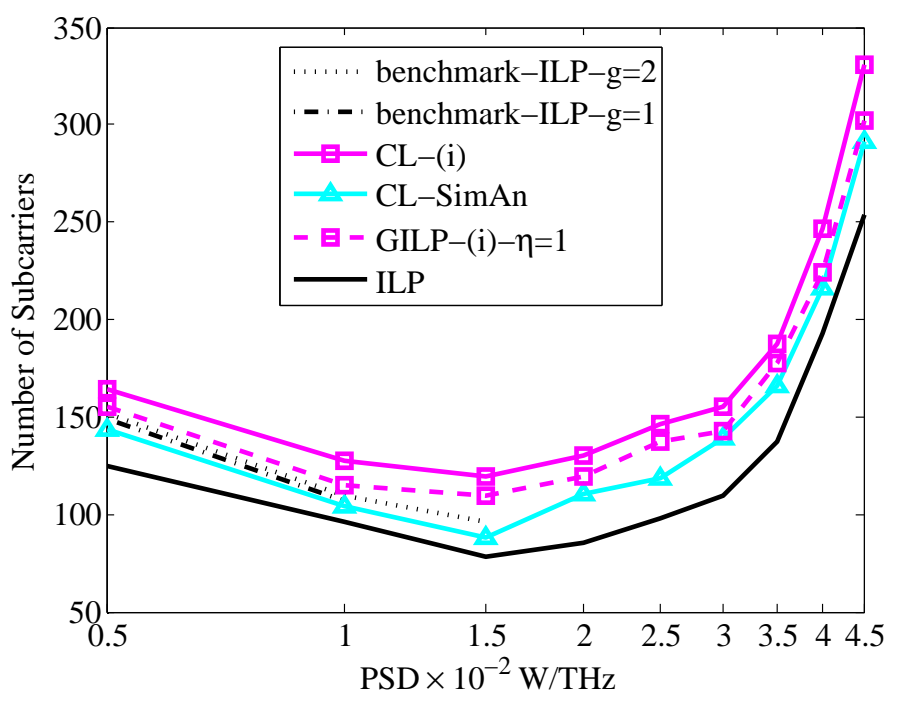

(a)

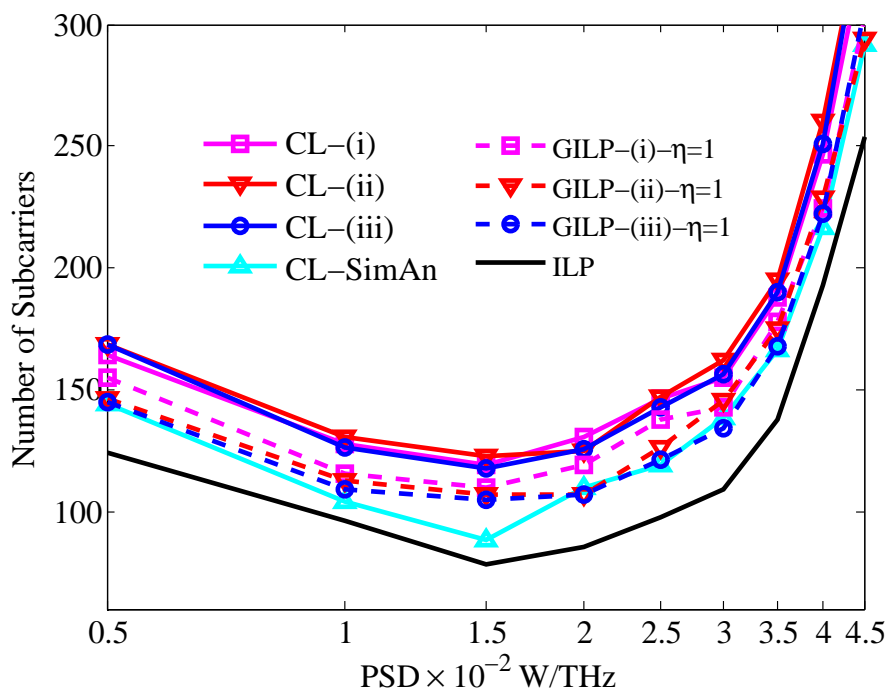

(b)

Fig. 5: The bandwidth (average of maximum allocated subcarrier indexes) vs. PSD ( $G$ in Table I) for the bit rate requirement of each connection is between $1250 \mathrm{Gbps}$ and 3750 Gbps in the 6-node network. (a) Main algorithms compared with benchmarks. (b) Proposed algorithms, including the sorting policies.

compared to the RMSA-SimAn with $g=1$ when $G=0.01$ W/THz. Also, CL-(iii) has better performance than CL-(i) and CL-(ii), since policy (iii) takes the bit rate requirement and the path distance into account.

\section{Conclusions}

In this paper, we investigated the nonlinear impairment aware routing and spectrum allocation problem in elastic networks based on Nyquist wavelength division multiplexing (NWDM) and optical orthogonal frequency division multiplexing (OOFDM). Simulation results indicate that, for the Deutsche Telekom network, the proposed algorithms outperform transmission reach based state-of-the-art methods up to $31 \%$ in terms of bandwidth under low bit rate requirements and up to $22 \%$ under high bit rate requirements. Furthermore, the proposed ILP optimization achieves 


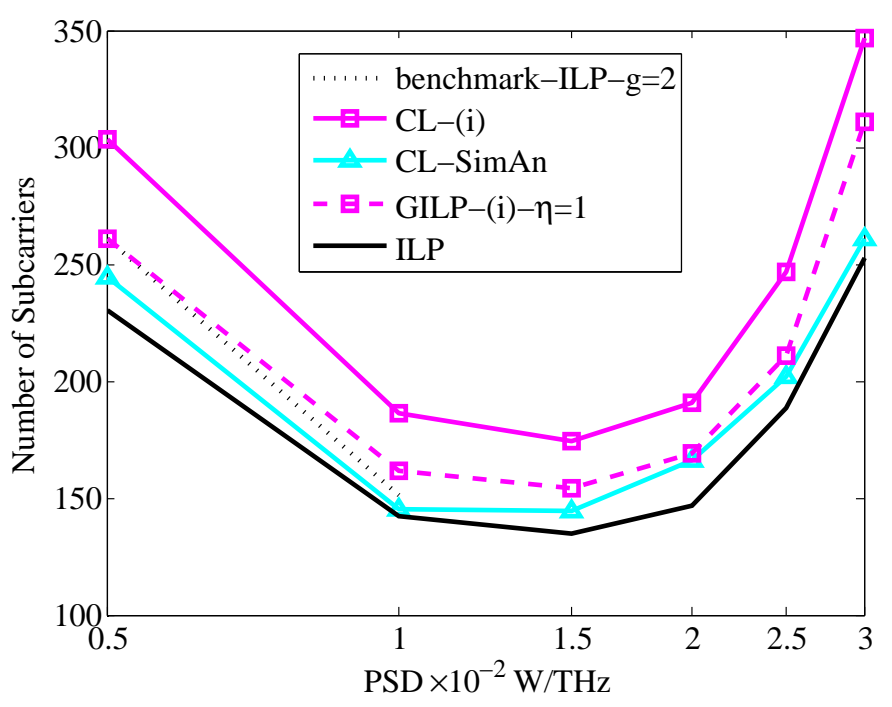

(a)

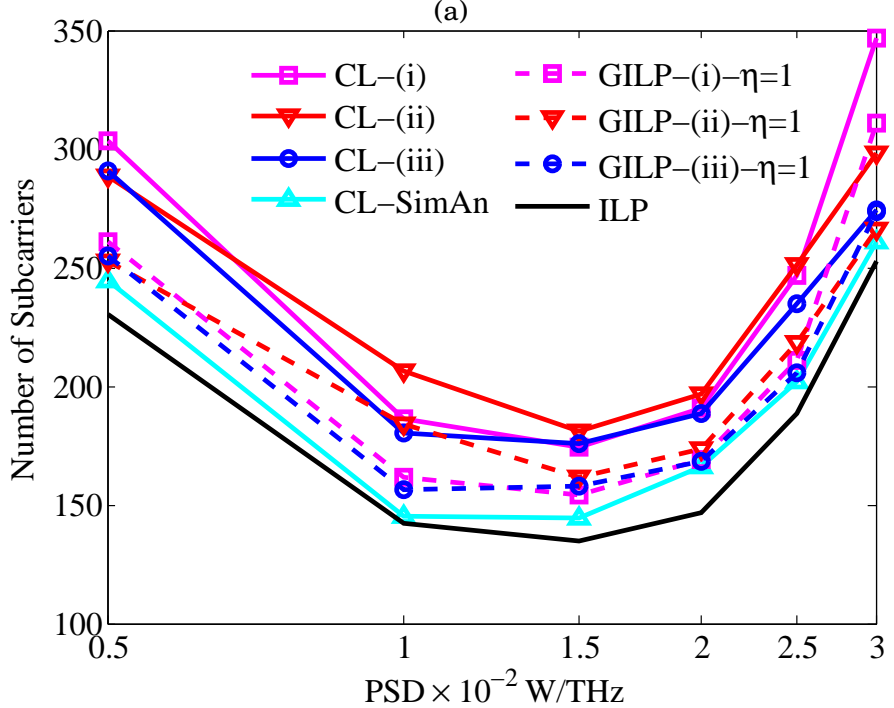

Fig. 6: The bandwidth (average of maximum allocated subcarrier indexes) vs. PSD ( $G$ in Table I) for the bit rate requirement of each connection is between $1250 \mathrm{Gbps}$ and 3750 Gbps in the 6-node network with doubled link length. (a) Main algorithms compared with benchmarks. (b) Proposed algorithms, including the sorting policies.

better performance for both uniform (up to $22 \%$ bandwidth reduction) and a non-uniform distribution (up to $13 \%$ bandwidth reduction) traffic demand in a 6-node network. In addition, it is shown that nonlinear impairments should be considered in the optimization problem to guarantee the quality of transmission and avoid infeasible solutions. When the differences of link lengths and differences of bit rate requirements for connections are large, it is better to utilize a sorted connection allocation order which takes into account both bit rate requirement and path length. The simulation results also show that the optimum power spectrum density (the power spectrum density value that achieves the lowest bandwidth allocation) decreases as the link length in the network increases and as the bit rate requirement of each connection increases, since either of the increases results in more nonlinear impairments.
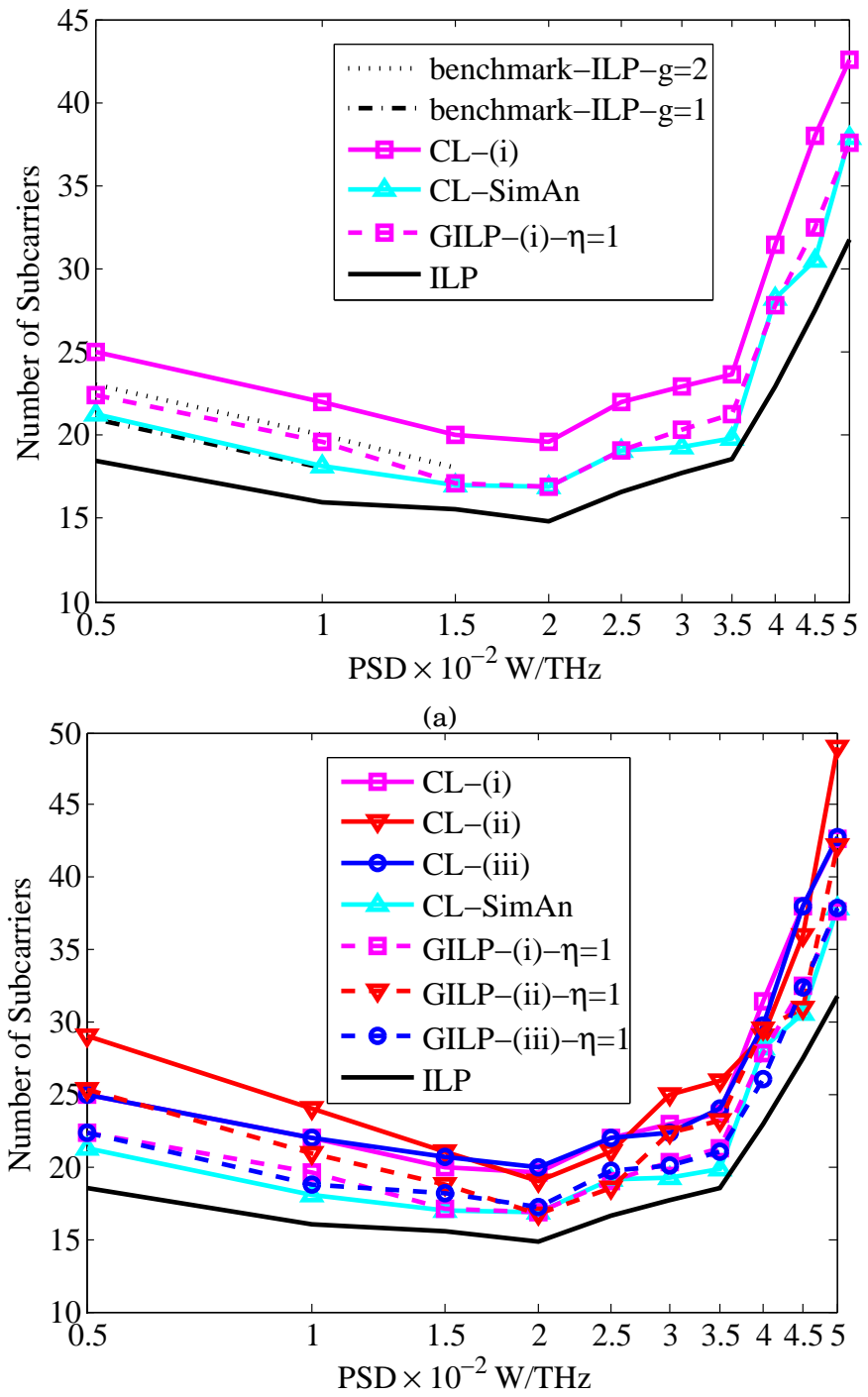

Fig. 7: The bandwidth (average of maximum allocated subcarrier indexes) vs. PSD ( $G$ in Table I) for nonuniform traffic demands in the 6-node network. (a) Main algorithms compared with benchmarks. (b) Proposed algorithms, including the sorting policies.

\section{REFERENCES}

[1] I.B. Djordjevic and B. Vasic, "Orthogonal frequency division multiplexing for high-speed optical transmission," Opt. Expr., vol. 14, no. 9, pp. 3767-3775, May 2006.

[2] M. Jinno, H. Takara, B. Kozicki, Y. Tsukishima, Y. Sone and S. Matsuoka, "Spectrum-efficient and scalable elastic optical path network: architecture, benefits, and enabling technologies," IEEE Commun. Mag., vol. 47, no. 11, pp. 66-73, Nov. 2009.

[3] R. Casellas, R. Munoz, J.M. Fabrega, M.S. Moreolo, R. Martinez, Lei Liu, T. Tsuritani and I. Morita, "GMPLS/PCE control of flexigrid DWDM optical networks using CO-OFDM transmission," IEEE /OSA J. Opt. Commun. Netw., vol. 4, no. 11, pp. B1-B10, Nov. 2012.

[4] F. Cugini, G. Meloni, F. Paolucci, N. Sambo, M. Secondini, L. Gerardi, L. Poti and P. Castoldi, "Demonstration of flexible optical network based on path computation element," IEEE/OSA J. Lightw. Technol., vol. 30, no. 5, pp. 727-733, Mar. 2012.

[5] M. Jinno, B. Kozicki, H. Takara, A. Watanabe, Y. Sone, T. Tanaka, and A. Hirano, "Distance-adaptive spectrum resource allocation in spectrum-sliced elastic optical path network," IEEE Commun. Mag., vol. 48, no. 8, pp. 138-145, Aug. 2010.

[6] X. Wan, N. Hua, and X. Zheng, "Dynamic routing and spectrum 


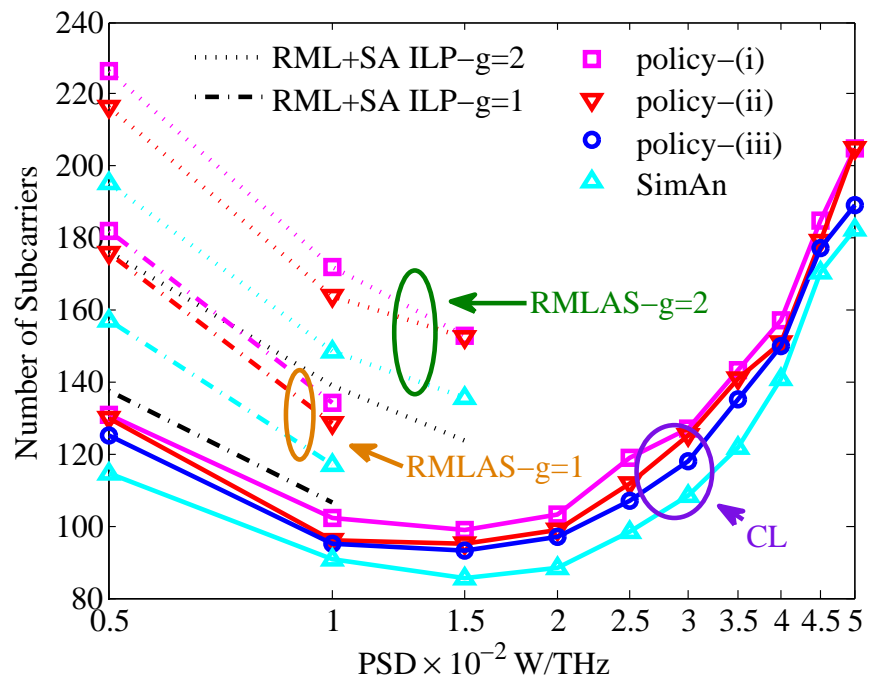

Fig. 8: The bandwidth (average of maximum allocated subcarrier indexes) vs. PSD ( $G$ in Table I) for the bit rate requirement of each connection is between $312.5 \mathrm{Gbps}$ and $625 \mathrm{Gbps}$ in the 14-node DT network for benchmark and CL heuristic with policies (i), (ii), (iii), and simulated annealing.

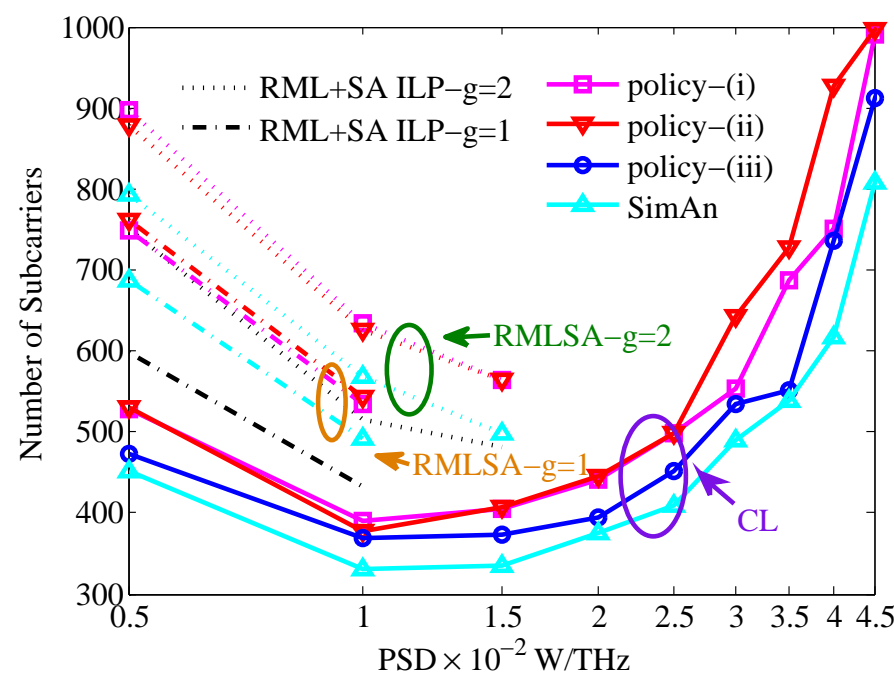

Fig. 9: The bandwidth (average of maximum allocated subcarrier indexes) vs. PSD ( $G$ in Table I) for the bit rate requirement of each connection is between $1250 \mathrm{Gbps}$ and $3750 \mathrm{Gbps}$ in the 14-node DT network for benchmark and CL heuristic with policies (i), (ii), (iii), and simulated annealing.

assignment in spectrum-flexible transparent optical networks," IEEE /OSA J. Opt. Commun. Netw., vol. 4, no. 8, pp. 603-613, Aug. 2012.

[7] Z. Zhu, W. Lu, L. Zhang, and N. Ansari, "Dynamic service provisioning in elastic optical networks with hybrid single-/multipath routing," IEEE/OSA J. Lightw. Technol., vol. 31, no. 1, pp. 15-22, Jan. 2013.

[8] X. Wang, K. Kuang, S. Wang, S. Xu, H. Liu, and G.N. Liu, "Dynamic routing and spectrum allocation in elastic optical networks with mixed line rates," J. Opt. Commun. Netw., vol. 6, no.12, pp. 1115-1127, Dec. 2014.

[9] K. Christodoulopoulos, I Tomkos, and E. Varvarigos, "Elastic bandwidth allocation in flexible OFDM-based optical networks," IEEE/OSA J. Lightw. Technol., vol. 29, no. 9, pp. 1354-1366, May 2011.

[10] K. Christodoulopoulos, P. Soumplis and E. Varvarigos, "Planning flexible optical networks under physical layer constraints," IEEE / OSA J. Opt. Commun. Netw., vol. 5, no. 11, pp. 1296-1312, Nov. 2013.
[11] H. Beyranvand and J.A. Salehi, "A quality-of-transmission aware dynamic routing and spectrum assignment scheme for future elastic optical networks," IEEE/OSA J. Lightw. Technol., vol. 31, no. 18, pp. 3043-3054, Sept. 2013.

[12] P. Kulkarni, A. Tzanakaki, C. Mas Machuca, and I. Tomkos, "Benefits of Q-factor based routing in WDM metro networks," in Proc. ECOC, Glasgow, U.K., Sept. 2005.

[13] X. Yang, and B. Ramamurthy, "Dynamic routing in translucent WDM optical networks the intradomain case," IEEE/OSA J. Lightw. Technol., vol. 23, no. 3, pp. 955-971, Mar. 2005.

[14] J. He, M. Brandt-Pearce and S. Subramaniam, "Optimal RWA for static traffic in transmission-impaired wavelength-routed networks," IEEE Communications Letters, vol. 12, no. 9, pp. 693695 , Sept. 2008

[15] A. Marsden, A. Maruta and K. Kitayama, "Routing and wavelength assignment encompassing FWM in WDM lightpath networks," in Proc. ONDM, Vilanova i la Geltru, Spain, Mar. 2008.

[16] J. Zhao, S. Subramaniam, and M. Brandt-Pearce, "Cross-layer RWA in translucent optical networks," in Proc. IEEE ICC, Ottawa, Canada, June 2012.

[17] D.J. Ives, P. Bayvel, and S.J. Savory, "Physical layer transmitter and routing optimization to maximize the traffic throughput of a nonlinear optical mesh network," in Proc. ONDM, Stockholm, Sweden, May 2014.

[18] D.J. Ives, A. Lord, P. Wright, and S.J. Savory, "Quantifying the impact of non-linear impairments on blocking load in elastic optical networks," in Proc. OFC/NFOEC, Mar. 2014.

[19] S. Fujii, Y. Hirota, H. Tode, and K. Murakami, "On-demand spectrum and core allocation for reducing crosstalk in multicore fibers in elastic optical networks," IEEE/OSA J. Opt. Commun. Netw., vol. 6, no. 12, pp. 1059-1071, Dec. 2014.

[20] J. Zhao, H. Wymeersch, and E. Agrell, "Nonlinear impairment aware resource allocation in elastic optical networks," in Proc. OFC, M2I.1, Los Angeles, March 2015.

[21] P. Johannisson and E. Agrell, "Modeling of nonlinear signal distortion in fiber-optic networks," IEEE/OSA J. Lightw. Technol., vol. 32, no. 23, pp. 4544-4552, Dec. 2014.

[22] Y. Wang, X. Cao, and Y. Pan, "A Study of the Routing and Spectrum Allocation in Spectrum-sliced Elastic Optical Path Networks", IEEE Infocom, April 2011.

[23] A. Cai, G. Shen, L. Peng, and M. Zukerman, "Novel NodeArc Model and Multiiteration Heuristics for Static Routing and Spectrum Assignment in Elastic Optical Networks," IEEE / OSA J. Lightw. Technol., vol. 31, no. 21, pp. 3402-3413, Nov. 2013.

[24] J. Y. Yen, "Finding the K shortest loopless paths in a network," Manage. Sci., vol. 17, no. 11, pp. 712-716, July 1971.

[25] Recommendation ITU-T G.694.1, 2012.

[26] D. Hillerkuss, R. Schmogrow, M. Meyer, S. Wolf, M. Jordan, P. Kleinow, N. Lindenmann, P. Schindler, A. Melikyan, X. Yang, S. Ben-Ezra, B. Nebendahl, M. Dreschmann, J. Meyer, F. Parmigiani, P. Petropoulos, B. Resan, A. Oehler, K. Weingarten, L. Altenhain, T. Ellermeyer, M. Moeller, M. Huebner, J. Becker, C. Koos, W. Freude, and J. Leuthold, "Single-Laser 32.5 Tbit/s Nyquist WDM Transmission," IEEE/OSA J. Opt. Commun. Netw., vol. 4, no. 10, pp. 715-723, Oct. 2012.

[27] D. Ives, P. Bayvel, and S. Savory, "Adapting Transmitter Power and Modulation Format to Improve Optical Network Performance Utilizing the Gaussian Noise Model of Nonlinear Impairments", IEEE/OSA J. Lightw. Technol., vol. 32, no. 21, pp. 34853494, Nov. 2014 